\title{
THEORETICAL ANALYSIS ON EFFICIENT MICROWAVE WARMING OF HUMAN BLOOD
}

\author{
S. Kumari ${ }^{1}$, S. K. Samanta ${ }^{1}$, B. Saxena ${ }^{1}$ \\ ${ }^{1}$ Indian Institute of Technology Patna, Bihta, Patna-801103, India \\ sushma.pcb16@iitp.ac.in; sksamanta@iitp.ac.in; brijsaxena1994@gmail.com
}

\begin{abstract}
:
To study the thermal impact of microwave irradiation on the human blood, a meticulous analysis has been performed with the aim of rapid generation of uniformly warmed blood assumed as a 2D cylinder. The analysis preliminarily involves the comparison of average microwave power absorption by the sample for various sample sizes on being subjected to two different irradiation schemes i.e. lateral and radial. The Galerkin finite element analysis has been applied to simultaneously solve the Maxwell's equation and heat transfer equation using pertinent boundary conditions to study the thermal effect of the microwave interaction with blood sample. Prelusive analysis of average power absorption and the subsequent detailed analysis of the heating rate and thermal non-uniformity through the plots of average temperature and temperature difference against time, respectively, have led to selection of optimum heating strategies for various blood samples corresponding to suitably selected sizes. Considering the frequency of $2450 \mathrm{MHz}$ and intensity of $1 \mathrm{~W} . \mathrm{cm}^{-2}$, the lateral irradiation is found to be more favorable for samples having moderate or large diameter, whereas the radial irradiation is found to be more suitable for small samples. Considering all the aspects, the present work recommends an efficient way for enhanced microwave assisted heating of human blood samples (2D cylindrical geometry) with known or measurable dielectric properties.
\end{abstract}

Keywords: Microwave, Human blood, Optimum heating, Thermal runaway, 2D cylinders

\section{Introduction:}

The microwave is a non-ionizing electromagnetic irradiation with the feature of volumetric processing $^{1-3}$. The frequency range of a microwave irradiation lies between $300 \mathrm{MHz}$ to 300 $\mathrm{GHz}$ in the electromagnetic spectrum. The interaction of microwave irradiation with a dielectric substance depends upon its factor of dielectric constant and loss values ${ }^{2}$. The value of dielectric loss determines the quantity of conversion of the electromagnetic energy to heat energy on interaction. The thermal effect of microwave irradiation has gained attention in different processing industries such as food, pharmaceutical, ceramics, polymer, medical sciences and many more ${ }^{2,4-6}$. The diverse applications of microwave in the field of medical science have always been the topic of extensive investigation from decades and have both therapeutic and diagnostic utility ${ }^{4-6}$.

The importance of warming of pre-transfusion blood has been a topic of great investigation ${ }^{7,8}$. The prominence of warming was accepted after several investigations demonstrated the complications of cold blood transfusion such as hemorrhagic shocks, ventricular fibrillation, etc $^{7-9}$. These studies highly favored the need to research and investigate all the aspects of warming of pre-transfusion blood. The human blood needs to be warmed rapidly taking in cogitation the preservation of its sterility and quality by employing microwave assisted warming technique ${ }^{10}$. The microwave assisted warming of human blood has been a suggested alternative in the category of dry technique of blood warming 8,11 . 
Although, the microwave warming of human blood started decades back, however the procedure was not widely accepted owing to the reports of causalities due to the transfusion of microwave warmed denatured blood ${ }^{12-14}$. So, the aim of this study is to perform a mathematical analysis using the information available in present literature about the dielectric properties of blood and analyze precisely the exact heating time and strategy for known sample size and ambience of the system ${ }^{15-17}$.

Microwave blood warmers drew two major stipulations for the acceptance of microwave assisted blood warming technique: The decimation of the occurrence of over-heating and the elimination of any other attributes of microwave which would damage the cell functionality ${ }^{18,19}$. So, they suggested circumvention of overheating to avoid any damage by microwave warming and the need of further detailed investigation to make it a clinically useful tool ${ }^{18,19}$.

In this work, a thermal analysis has been carried out which involves the comparison of heating effects of microwave irradiation with frequency $(f)=2450 \mathrm{MHz}$ and intensities $\left(I_{0}\right)$ $=1,1.5$ and $2 \mathrm{~W} \cdot \mathrm{cm}^{-2}$, on human blood samples of various sizes and on being subjected to two different irradiation schemes i.e. lateral and radial. The exploration of heating rate and thermal non-uniformity through the plots of average temperature and temperature difference against time, respectively, will lead to the selection of the optimum heating strategies for various blood samples corresponding to suitably selected sizes.

\section{Theoretical formulations:}

\section{Power and temperature calculations:}

The evaluation of the extent of absorption of microwave irradiation by the blood sample has been performed through the electromagnetic field equation. The evaluation aids the calculation of temperature distribution inside the sample when subjected to different modes of irradiation i.e. lateral and radial. The lateral irradiation mode is the situation designed with an assumption of a stationary sample in a uniform plane wave having interaction from one direction (left in this study), whereas radial irradiation mode assumes the sample to rotate with the surety of uniform interaction from all the directions.

The electromagnetic field representing microwave irradiation consists of electric and magnetic fields which are perpendicular to each other and have the variation of the magnitude in the direction of propagation i.e. $\mathrm{z}$ direction (see Fig. 1). The electric field propagation equation is represented by the Maxwell's equation ${ }^{1}$ i.e.

$\nabla^{2} E_{x}+\kappa^{2} E_{x}=0$

where, $E_{x}$ is the electric field which varies in $\mathrm{y}-\mathrm{z}$ plane and $\kappa$, the propagation constant, depends on dielectric constant $\kappa^{\prime}$, dielectric loss $\kappa^{\prime \prime}$ of the sample, frequency $f$ of the irradiation and speed of light $c$. The relation is given as:

$\kappa=\frac{2 \pi f}{c} \sqrt{\kappa^{\prime}+i \kappa^{\prime \prime}}$

Further, the calculation of the change in the phase of the wave and the field intensity decay rate is carried out using the relation between wavelength $\left(\lambda_{s}\right)$ and penetration depth $\left(\delta_{p}\right)$ with the dielectric properties of the sample, respectively as ${ }^{1}$ : 


$$
\begin{aligned}
& \lambda_{s}=\frac{c}{\sqrt{\frac{\kappa^{\prime} f^{2}\left(\left(1+\tan ^{2} \delta\right)^{1 / 2}+1\right)}{2}}} \\
& \delta_{p}=\frac{c / \pi}{\sqrt{\frac{\kappa^{\prime} f^{2}\left(\left(1+\tan ^{2} \delta\right)^{1 / 2}-1\right)}{2}}}
\end{aligned}
$$

where, $\tan \delta=\frac{\kappa^{\prime \prime}}{\kappa^{\prime}}$ represents the loss tangent.

The dissipation of power in the sample is evaluated using the Poynting theorem and can be given as:

$\nabla \cdot S=-\frac{1}{2} \omega \varepsilon_{0} \kappa^{\prime \prime} E_{x} \cdot E_{x}^{*}+i \omega\left(\frac{\mu_{0}}{2} H_{y} \cdot H_{y}^{*}+\frac{\varepsilon_{0} \kappa^{\prime}}{2} E_{x} \cdot E_{x}^{*}\right)$

The real part of the equation gives the power dissipation and the imaginary part gives the stored electric and magnetic power. So, the local power dissipation is ${ }^{1}$ :

$P=-\operatorname{Re}(\nabla \cdot S)=\frac{1}{2} \omega \varepsilon_{0} \kappa^{\prime \prime} E_{x} \cdot E_{x}^{*}$

The time dependent heat equation for the system involves a heat conduction equation with heat source term due to microwave power $(P)$ can be given as: $\rho C_{p} \frac{\partial T}{\partial t}=\nabla \cdot(k \nabla T)+P$

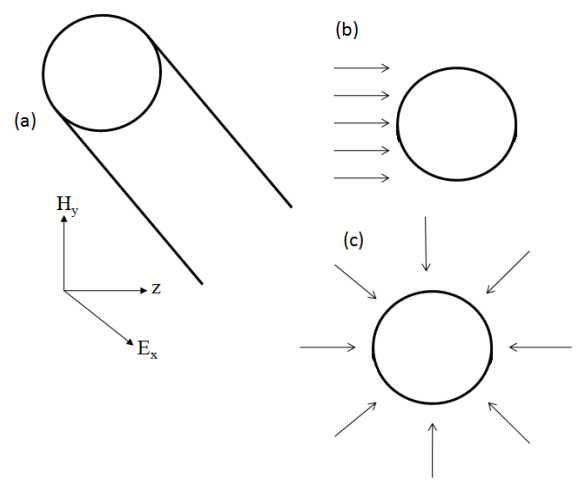

Fig. 1. Schematic illustrations of 2D human blood cylinders incident with (a) uniform plane waves, (b) lateral irradiation and (c) radial irradiation.

The insulation condition (no flux condition) has been used for outer surface as the boundary condition.

\section{Calculation strategies:}

The simultaneous evaluation of the heat equation and electric field equation with pertinent boundary conditions is performed through Galerkin finite element method. The time domain is discretized using Crank-Nicholson method, and Newton Raphson method is used to solve the non-linear residual equations with the first time step of $1 * 10^{-4}$ seconds and subsequently 0.1 seconds. The initial temperature of the blood sample and the ambient temperature for the analysis are considered to be $273 \mathrm{~K}$ and $300 \mathrm{~K}$, respectively. 


\section{Results and discussion:}

The thermal effect of microwave irradiation on human blood samples without any additives has been investigated. The samples have been assumed to be cylindrical in shape and have been subjected to microwave irradiation by lateral and radial mode of incidences of the irradiation. A prolegomenon investigation on the average microwave power the human blood absorbed for a given sample size is performed. The plot obtained revealed several peak points which are as a result of constructive interferences between transmitted and reflected waves of the microwave irradiation (see Fig. 2). The peak points of average power absorption at the emphasized conditions of lateral and radial irradiations have been further analyzed to study the spatial distribution of power and temperature.

\section{Microwave warming of human blood with intensity, $I_{0}=1 \mathrm{~W} \cdot \mathrm{cm}^{-2}$}

The peaks observed in the plot are caused due to constructive interference of the propagating microwave and show the maximum values of the average power absorbed by the human blood. The observation points are selected on the basis of either of the irradiation techniques achieving a maxima in the average power absorption observed. The $1^{\text {st }}$ observation point (OP) considering the initial sample size, $d_{c}>1 \mathrm{~cm}$ is observed at $d_{c}=1.154 \mathrm{~cm}(O P: 1)$ for lateral and radial irradiation with average power of $\mathrm{P}_{\mathrm{av}}=2.004$ and $1.612 \mathrm{~W} . \mathrm{cm}^{-3}$, respectively. The next OP occurs at $\mathrm{d}_{\mathrm{c}}=1.894 \mathrm{~cm}(\mathrm{OP}: 2)$ with average power of $\mathrm{P}_{\mathrm{av}}=0.687$ and $1.765 \mathrm{~W} . \mathrm{cm}^{-3}$ for lateral and radial irradiations, respectively. The third observation point for lateral and radial irradiation is obtained at $\mathrm{d}_{\mathrm{c}}=3.378 \mathrm{~cm}(\mathrm{OP}: 3)$ with corresponding average power absorption $\mathrm{P}_{\mathrm{av}}=0.264$ and $0.408 \mathrm{~W} . \mathrm{cm}^{-3}$, respectively. The OP: 1 exhibited a higher value of average power absorption for lateral heating scheme whereas the OP: 2 and OP: 3 depict the radial heating scheme to exhibit higher value of average power absorptions.

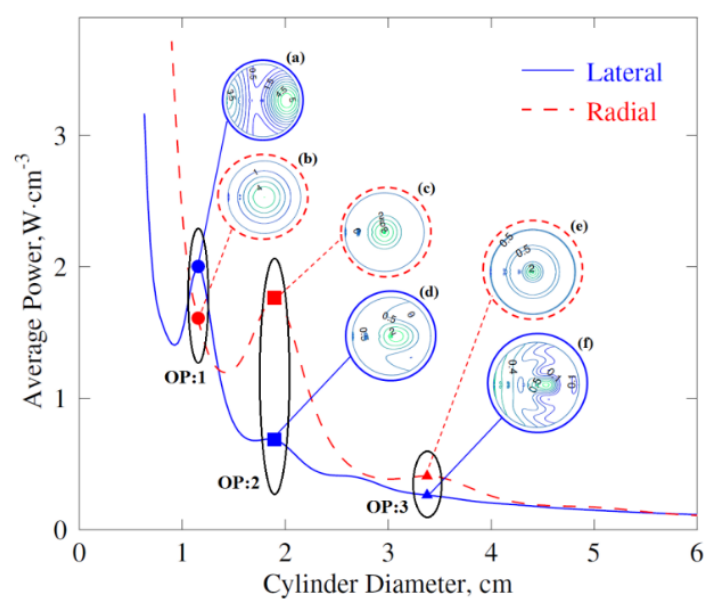

Fig. 2. The representation of average power absorption $\left(\mathrm{W} . \mathrm{cm}^{-3}\right)$ in the human blood sample with respect to sample size $(\mathrm{cm})$ during microwave assisted warming at frequency of $2450 \mathrm{MHz}$ and intensity of $1 \mathrm{~W} . \mathrm{cm}^{-2}$. The inset is the representation of the power distribution inside the cylindrical sample (a) for lateral heating at $\mathrm{d}_{\mathrm{c}}=1.154 \mathrm{~cm}$, (b) for radial heating at $d_{c}=1.154 \mathrm{~cm},(\mathbf{c})$ for radial heating at $\mathrm{d}_{\mathrm{c}}=1.894 \mathrm{~cm},(\mathbf{d})$ for lateral heating at $d_{c}=1.894 \mathrm{~cm}$, (e) for radial heating at $d_{c}=3.378 \mathrm{~cm}$ and (f) for lateral heating at

$$
\mathrm{d}_{\mathrm{c}}=3.378 \mathrm{~cm} \text {. }
$$


The details of distribution of power absorption need to be further analyzed extensively due to the sample being prone to denaturation on exposure to high power and non-uniform heating. The OPs are further analyzed in detail regarding the uniformity of power and temperature dissemination along with rate of heating. The insets in the Fig. 2 show the spatial distribution of absorbed power by the human blood with respect to the size of the sample subjected to microwave irradiation in both lateral and radial modes. The power distribution contour for lateral irradiation at OP: 1 shows two cruxes of high power absorption on either sides of the sample with left at $3.5 \mathrm{~W} . \mathrm{cm}^{-3}$ and right side at $5 \mathrm{~W} \cdot \mathrm{cm}^{-3}$ (see Fig. 2(a)). These are the possible causes of constructive interferences of the waves inside the sample being subjected to the microwave irradiation. The power at the centre of the sample ranges from 1.5 to $0.5 \mathrm{~W} . \mathrm{cm}^{-3}$ depicting low power absorption. Moving on to OP: 2 and being consistent with lateral mode of irradiation, the power absorbed by the sample is observed to shift towards the centre and reduce to $2 \mathrm{~W} \cdot \mathrm{cm}^{-3}$ (see Fig. 2 (d)). The OP: 3 for the lateral irradiation exhibits thoroughly concentrated average power absorption at the centre with higher values of $0.5 \mathrm{~W} . \mathrm{cm}^{-3}$ (see Fig. 2 (f)). On studying the radial mode of irradiation at the 3 OPs, a single high power absorption crux residing at the centre of the sample and reduce when traversing towards the edge. The value of power varies from $4 \mathrm{~W} . \mathrm{cm}^{-3}, 8 \mathrm{~W} . \mathrm{cm}^{-3}$ and 2 W.cm ${ }^{-3}$ for OP: 1, 2 and 3, respectively (see Fig. 2 (b), (c) and (e)).

The Fig. 3(a) is the surface contour exhibiting the distribution of temperature on being exposed to the microwave irradiation for 80 seconds with $I_{0}=1 \mathrm{~W} \cdot \mathrm{cm}^{-2}$. The lateral mode of irradiation at OP: 1 displays a maximum temperature of $335 \mathrm{~K}$ on the right side of the sample and minimum temperature of $305-310 \mathrm{~K}$ and radial mode a central maxima of about $318 \mathrm{~K}$. The OP: 2 manifests a single central maxima of $296 \mathrm{~K}$ and minima of $282 \mathrm{~K}$ towards the right sided outer edge on lateral and a central maxima of $340 \mathrm{~K}$ on radial microwave exposure. The lateral irradiation mode at OP: 3 displays a left inclined maxima of around $283 \mathrm{~K}$ whereas the radial mode presents a central maxima of $290 \mathrm{~K}$.

(a)
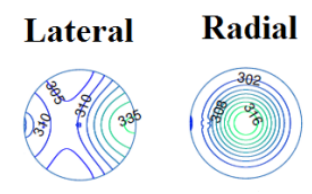

OP:2
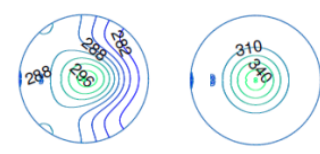

OP:3
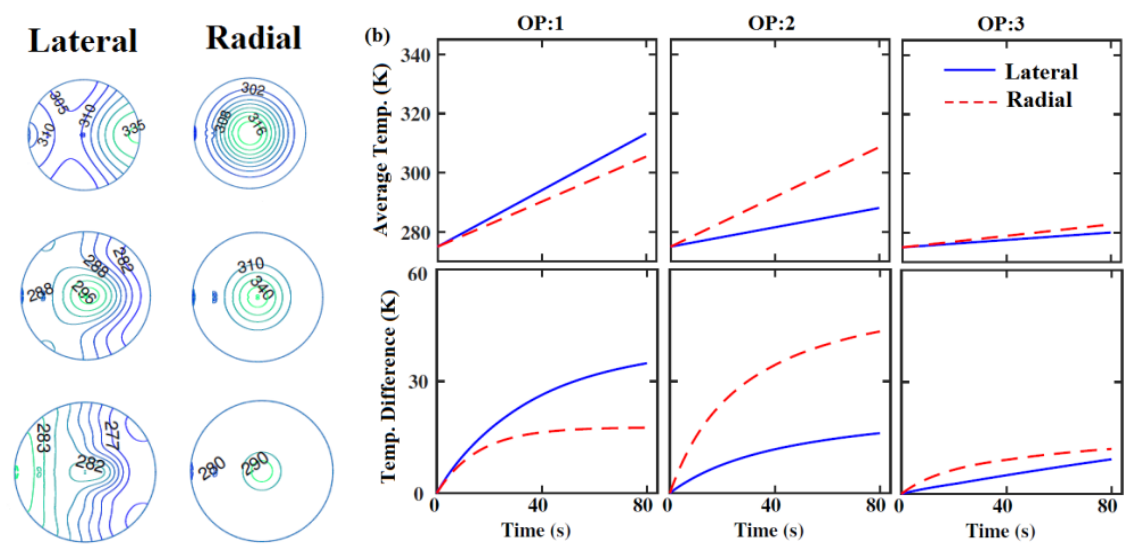

Fig. 3. (a) Spatial representation of thermal mapping inside the sample during microwave assisted warming at frequency of $2450 \mathrm{MHz}$ and intensity of $1 \mathrm{~W} \cdot \mathrm{cm}^{-2}$ for 80 seconds. (b)

Average temperature (K) in the human blood vs. time (s) [upper panel]; Temperature difference $(\mathrm{K})$ in the human blood vs. time (s) [lower panel].

The Fig. 3 (b) gives the plot of average temperature (K) in the blood sample with respect to time (s) measuring the heating rate and the temperature difference $(\mathrm{K})$ in blood sample with respect to time (s) representing the non-uniformity of the temperature distribution or the thermal runaway occurring in the sample. The optimum heating strategy out of either lateral 
or radial irradiation mode for a chosen set of sample size, intensity and exposure duration is set on the criteria of high heating rate and low thermal non-uniformity. The OP: 1 put forth the lateral irradiation mode having a slightly higher heating rate with a very high thermal non-uniformity making it the undesired heating strategy. However, lateral irradiation is preferred for OP: 2 due to very high thermal runaway even with higher heating rate owing to the delicate nature of blood samples and have identical situation for OP: 3. A similar analysis is performed to the samples at different time steps with different input intensities for understanding the pattern for microwave warming for different sizes of human blood sample.

\section{Conclusions:}

The mathematical analysis was performed with the objective to understand the thermal effects of microwave irradiation on very delicate human blood samples assumed as a $2 \mathrm{D}$ cylindrical geometry. The study is performed by solving the equation of electromagnetic wave propagation along with the equation of energy balance and pertinent boundary conditions. The sample was subjected to two different modes of interaction of the microwave with the sample i.e. lateral and radial. The initial analysis put forward the change in nature of average power absorption occurring at different sample sizes due to the resonance effects of the electromagnetic wave interaction. The peaks observed are chosen as observation points for detailed analysis about the spatial distribution of power and temperature inside the sample. The microwave warming of human blood at $2450 \mathrm{MHz}$ and $1 \mathrm{~W} . \mathrm{cm}^{-2}$ gives the plot of average power with respect to the sample size exhibiting the lateral mode to with higher average power absorption for small sizes samples. However, the average power is more absorbed through the radial irradiation mode for medium to large sized samples. Further spatial analysis of the temperature and power is performed displaying the number of maxima obtained along with its position. The plot of average temperature and temperature difference with respect to time leads to the selection of the condition of high heating rate and low thermal non-uniformity as the parameter to decide the optimum heating strategy for either lateral or radial irradiation mode. The OP: 1 indicates the radial mode and the OP: 2 and 3 indicate the lateral mode of irradiation as the optimum strategy for the microwave blood warming. In conclusion we can say that the efficient warming technique is dependent on the dielectric property of the sample, exposure duration, the property of the wave and many more. The analysis acts as a guideline for development of a safe and rapid warming technique for human blood or other delicate crystalloid fluids using microwave irradiation with consideration to all the strategies and sample properties.

\section{References}

1. Ayappa KG, Davis HT, Davis EA, Gordon J. Aiche J. 1992;38:1577-92.

2. Samanta SK, Basak T. Food Res Int. 2010;43:148-66.

3. Samanta SK, Basak T. Food Res Int. 2009;42:1337-50.

4. Vrba J. Electromagn Biol Med. 2005;24:441-8.

5. Rosen A, Stuchly MA, Vorst A Vander. IEEE Trans Microw Theory Tech. 2002;50:963-74.

6. Yang WJ, Mochizuki S, Yang PPT. J Mech Med Biol. 2002;2:53-65.

7. Boyan CP. Ann Surg. 1964;160:282-6.

8. Iserson K V, Huestis DW. Transfusion. 1991;31:558-71.

9. Restall CJ, Leonard PF, Taswell HF, Holaday RE. Anesth Analg. 1967;46:625-8.

10. Smith CE, Wagner K. Int TraumaCare. 2008;18:71-9.

11. Campbell NL, Drewe J. IEEE MTT-S Int. 1981;479-81.

12. Arens JF, Leonard GL. JAMA. 1971;218:1045-6. 
13. Hirsch J, Menzebach A, Welters ID, Dietrich GV, Katz N, Hempelmann G. Clin Chem. 2003;49:792-9.

14. Linko K, Hynynen K. Acta Anaesthesiol Scand. 1979;23:320-8.

15. Takeda A, Takata K, Wang J, Fujiwara O. Meas 2011, Proceedings 8th Int Conf Smolenice, Slovakia. 2011;387-90.

16. Salahuddin S, O'Halloran M, Porter E, Farrugia L, Bonello J, Sammut C V, et al. IEEE Trans Dielectr Electr Insul. 2017;24:3283-9.

17. Rauf A. Int J Sci Environ Technol. 2013;2:1396-400.

18. Schwaitzberg SD, Allen MJ, Connolly RJ, Grabowy RS, Carr KL, Cleveland RJ. J Investig Surg. 1991;4:505-10.

19. Herron DM, Grabowy R, Connolly R, Schwaitzberg SD. J Trauma Acute Care Surg. 1997;43:9291364. 